\title{
Fast sequence evolution of Hox and Hox-derived genes in the genus Drosophila Sònia Casillas ${ }^{1}$, Bárbara Negre ${ }^{1,2}$, Antonio Barbadilla ${ }^{1}$ and Alfredo Ruiz*1
} Address: ${ }^{1}$ Departament de Genètica i de Microbiologia, Universitat Autònoma de Barcelona, 08193 Bellaterra (Barcelona), Spain and ${ }^{2}$ Department
of Zoology, University of Cambridge, Cambridge CB2 3EJ, UK

Email: Sònia Casillas - Sonia.Casillas@uab.es; Bárbara Negre -bn219@cam.ac.uk; Antonio Barbadilla - Antonio.Barbadilla@uab.es; Alfredo Ruiz* - Alfredo.Ruiz@uab.es

* Corresponding author

Published: 12 December 2006

BMC Evolutionary Biology 2006, 6:106 doi:10.1186/1471-2148-6-106
Received: 13 June 2006

Accepted: 12 December 2006

This article is available from: http://www.biomedcentral.com/I47I-2/48/6/106

(c) 2006 Casillas et al; licensee BioMed Central Ltd.

This is an Open Access article distributed under the terms of the Creative Commons Attribution License (http://creativecommons.org/licenses/by/2.0), which permits unrestricted use, distribution, and reproduction in any medium, provided the original work is properly cited.

\begin{abstract}
Background: It is expected that genes that are expressed early in development and have a complex expression pattern are under strong purifying selection and thus evolve slowly. Hox genes fulfill these criteria and thus, should have a low evolutionary rate. However, some observations point to a completely different scenario. Hox genes are usually highly conserved inside the homeobox, but very variable outside it.

Results: We have measured the rates of nucleotide divergence and indel fixation of three Hox genes, labial (lab), proboscipedia $(p b)$ and abdominal-A (abd-A), and compared them with those of three genes derived by duplication from Hox3, bicoid $(b c d)$, zerknüllt (zen) and zerknüllt-related (zen2), and 15 non-Hox genes in sets of orthologous sequences of three species of the genus Drosophila. These rates were compared to test the hypothesis that Hox genes evolve slowly. Our results show that the evolutionary rate of Hox genes is higher than that of non-Hox genes when both amino acid differences and indels are taken into account: $43.39 \%$ of the amino acid sequence is altered in Hox genes, versus $30.97 \%$ in non-Hox genes and $64.73 \%$ in Hox-derived genes. Microsatellites scattered along the coding sequence of Hox genes explain partially, but not fully, their fast sequence evolution.
\end{abstract}

Conclusion: These results show that Hox genes have a higher evolutionary dynamics than other developmental genes, and emphasize the need to take into account indels in addition to nucleotide substitutions in order to accurately estimate evolutionary rates.

\section{Background}

Hox genes are homeobox containing genes involved in the specification of regional identities along the anteroposterior body axis and, thus, play a fundamental role in animal development [1]. They encode transcription factors that regulate the expression of other genes downstream in the regulatory cascade of development and have been found in all metazoans, including flies, worms, tunicates, lampreys, fish and tetrapods. A particular feature of these genes is that they are usually clustered together in complexes and arranged in the chromosome in the same order as they are expressed along the anteroposterior body axis of the embryo $[2,3]$. Ten genes arranged in a single complex comprised the ancestral Hox gene complex of arthropods (HOM-C) [4-6]. However, at least three different HOM-C splits have occurred during the evolution of dip- 
tera [7-10], and several non-homeotic genes and other genes derived from ancestral Hox genes are interspersed among the Drosophila Hox genes.

The stability of Hox gene number and the conservation of Hox ortholog sequences prompted the notion that Hox proteins have not significantly diverged in function. However, it is now known that several arthropod Hox proteins have changed in sequence and/or function, including those encoded by Hox3 [11-13], fushi tarazu (ftz) [14], Ultrabithorax (Ubx) [15] and Antennapedia (Antp) [16]. In winged insects, including Drosophila, Hox3 and ftz lost their homeotic function, that is, their ability to transform the characteristics of one body part into those of another body part $[17,18]$, and their expression domains are no longer arranged along the anteroposterior axis of the embryo. Therefore, only eight Hox genes remain in these species [6]. Hox3 gained a novel extraembryonic function, and underwent two consecutive duplications that gave rise to bicoid $(b c d)$, zerknüllt (zen) and zerknüllt-related (zen2). The first duplication took place in the cyclorrhaphan fly lineage and gave rise to zen and bcd $[12,13]$. Afterwards, but before the Drosophila radiation, zen went through a second duplication that gave birth to zen2 [19]. Seemingly, $b c d$ and zen have specialized and perform separate functions in the establishment of the embryo's body plan: the maternal gene $b c d$ codes for an important morphogen that establishes anteroposterior polarity [20] and $z e n$ is a zygotic gene involved in dorsoventral differentiation [21]. zen2 has the same expression pattern of zen, although its function is unknown. Despite its high sequence divergence across species, it has been maintained for more than 60 Myr [19].

Hox proteins contain a highly conserved domain of 60 amino acids (coded by the homeobox) that binds DNA through a 'helix-turn-helix' structure. This motif is very similar in terms of sequence and structure to that of many DNA binding proteins. Functional comparisons of Hox orthologs have largely focused on their highly conserved homeodomain sequences and have demonstrated their functional interchangeability between species [22-26]. Hox-derived genes, although having lost their homeotic function, still retain the homeobox.

It has been shown that housekeeping genes, which are expressed in all cells and at all times, are under strong purifying selection and thus evolve slowly (e.g. histones, or genes involved in the cell cycle) $[27,28]$. Hox genes, on the contrary, are expressed early in development and have a complex regulated expression pattern. Mutations in such genes will on average have more deleterious fitness consequences than mutations occurring in genes expressed later on, because they may have cascading consequences for the later steps in development and thus may broadly alter the adult phenotype [29-31]. Therefore, we also expect Hox genes to be highly constrained and thus evolve slowly. In fact, Davis, Brandman, and Petrov [29] found a highly significant relationship between the developmental timing of gene expression and their nonsynonymous evolutionary rate: genes expressed early in development are likely to have a slower rate of evolution at the protein level than those expressed later. Surprisingly, the strongest negative relationship between expression and evolutionary rate occurred only after the main burst of expression of segment polarity and Hox genes in embryonic development, so these genes could be evolving differently from other developmental genes. However, only one segment polarity gene, wingless ( $w g$ ), and two Hox genes, Antp and abdominal-A (abd-A), were analyzed.

Furthermore, Marais et al. [32] found a negative correlation between evolutionary rate at the protein level (as measured by the number of nonsynonymous substitutions per nonsynonymous site, $d_{N}$ ) and intron size in Drosophila, likely due to a higher abundance of cis-regulatory elements in introns (especially first introns) in genes under strong selective constraints. We know from a previous study that the Hox genes used in this study contain a long intron replete with regulatory elements [19]. Therefore, we would expect these genes to be strongly constrained.

However, other studies seem to point to a completely different scenario. Developmental biologists noticed a long time ago that a large portion of the sequence of Hox proteins diverges so fast that it is difficult to align homologues from different arthropod classes [33]. In fact, nucleotide sequences outside the homeobox in labial (lab) and $U b x$ have been reported to diverge significantly $[8,15]$. These sequence differences may be neutral with respect to protein function or, more intriguingly, they could be involved in the functional divergence of Hox proteins and the evolutionary diversification of animals [15]. Moreover, Karlin and Burge [34] have shown that many essential developmental genes, including Hox genes, contain long microsatellites within their coding sequence (e.g. trinucleotide repeats that do not disrupt the open reading frame). The vast majority of these genes function in development and/or transcription regulation, and are expressed in the nervous system. Due to the particular mutation mechanism acting on these repetitive sequences by replication slippage $[35,36]$, microsatellites are subject to frequent insertions and deletions. Thus, these repetitive sequences could be responsible for a higher than expected evolutionary rate of Hox genes. However, and despite all the previous contributions, no quantification of the rates of nucleotide and indel evolution has been reported so far for a set of Hox genes. 
On the other hand, the origin by duplication and the functional evolution of Hox-derived genes suggest that they might be evolving fast at the sequence level as well. Duplicated genes are known to undergo a period of accelerated evolution where: they may degenerate to a pseudogene (pseudogenization), each daughter gene may adopt part of the functions of their parental gene (subfunctionalization), or they may acquire new functions (neofunctionalization) [37-40]. The only divergence estimate reported in a Hox-derived gene was calculated between two close species (D. melanogaster and D. simulans) in $b c d$ [41]. A recent study found an increased sequence polymorphism in bcd in comparison to zen, which was ascribed to a relaxation of selective constraint on this maternal gene resulting from sex-limited expression [42]. Therefore, $b c d$ is expected to evolve faster than zen under this model. The evolutionary rates of zen and zen2, however, have not been reported so far.

We have measured the rates of nucleotide substitution and indel fixation of three Hox genes, lab, proboscipedia $(p b)$ and $a b d-A$, and compared them with those of $b c d$, zen and zen2, which were derived by duplication from Hox3, and a sample of 15 non-Hox genes, in the genus Drosophila. These rates were compared to test the hypothesis that Hox genes, similar to other genes with complex expression patterns and that are essential in the early development, evolve slowly. We have also evaluated the contribution of the homeobox and the repetitive regions within Hox and Hox-derived genes to the evolutionary rates.

The sequences compared comprise all the complete genes available in D. buzzatii (representative of the Drosophila subgenus), and their orthologs in D. melanogaster and $D$. pseudoobscura (both species in the Sophophora subgenus). D. buzzatii belongs to the repleta species group, a group comprising $~ 100$ species that has been widely used as a model in studies of genome evolution, ecological adaptation and speciation. Negre et al. [19] have recently compared the genomic organization of the HOM-C complex in D. buzzatii to that of D. melanogaster and D. pseudoobscura, and studied the functional consequences of two HOM-C splits present in this species. When our study began, this was the largest set of orthologous Hox genes in species from both subgenera of the Drosophila genus, and this allowed the exploration of evolutionary rates throughout the Drosophila phylogeny. Due to the high divergence of Hox genes [8], the inclusion of more distant species outside the Drosophila genus (such as mosquito or honeybee) would probably not be appropriate for the estimation of genetic distances. Moreover, these species do not contain the Hox-derived genes studied here.

\section{Results \\ Nucleotide evolution of Hox, Hox-derived and non-Hox genes}

Nucleotide substitution parameters were calculated for the coding nucleotide alignments independently for each gene [see Additional file 1]. We then tested for differences between the three groups of genes (Hox, Hox-derived and non-Hox) (top section of Table 1) [see Additional file 2]. Our results showed that Hox-derived genes are evolving much faster and with less functional constraint than Hox and non-Hox genes. Differences among the three groups are significant for the number of nonsynonymous substitutions per nonsynonymous site, $d_{N}(P=0.022)$, and the level of functional constraint, $\omega(P=0.000)$ (see Methods). The gene zen 2 is the main gene responsible for the high values of nucleotide substitutions (both synonymous and nonsynonymous) in its group [see Additional file 1]. On the contrary, Hox and non-Hox genes have a similar number of nucleotide substitutions, $t(\mathrm{P}>0.1)$. However the level of functional constraint is even higher (lower $\omega$ ) in non-Hox genes than in Hox genes $(\omega=$ 0.04156 versus $\omega=0.06094$, respectively), although differences are only marginally significant $(P=0.063)$. Therefore, Hox genes do not seem to be evolving more slowly than other non-homeotic genes, despite their essential function in early development.

Then, we plotted $d_{N}$ and $\omega$ in sliding windows along the coding sequences of Hox and Hox-derived genes to see whether or not these parameters behave homogeneously along the sequence. Figure 1 shows that, in all genes except zen2, there is a substantial decrease of both $d_{N}$ and $\omega$ near the homeobox. zen 2 contains a rapidly evolving homeobox with high $\omega$ values. Contrarily, we have observed that peaks of $d_{N}$ tend to lie within repetitive regions (data not shown).

To control for a possible effect on the overall nucleotide evolution of both the homeobox and the repetitive regions (see Methods) of these Hox and Hox-derived genes, we tested again for differences among the three groups of genes excluding these regions. Removing the homeobox in Hox and Hox-derived coding sequences (second section of Table 1) elevated the number of nucleotide substitutions in these two groups, and decreased further their level of functional constraint. Again, differences among groups were significant for $d_{N}(\mathrm{P}=0.005)$ and $\omega(\mathrm{P}$ $=0.000)$, and the same tendency of the previous analysis with complete coding sequences was observed. In contrast, removing repetitive regions (third section of Table 1 ) decreased the number of nucleotide substitutions, especially in Hox genes, where all the genes in the group contain this type of region. Therefore, the elimination of repetitive regions slightly increases the difference between Hox and non-Hox genes in terms of nucleotide substitu- 
Table I: Mean nucleotide substitution parameters and ANOVAs for the three groups of genes.

\begin{tabular}{|c|c|c|c|c|c|}
\hline & & $t$ & $\boldsymbol{d}_{N}$ & $d_{S}$ & $\omega$ \\
\hline \multirow[t]{4}{*}{ Complete coding sequences } & Hox & 2.10917 & 0.15964 & 2.59066 & 0.06094 \\
\hline & Hox-derived & 3.86336 & 0.39380 & 4.27598 & 0.09226 \\
\hline & Non-Hox & 2.91160 & 0.15802 & 3.80668 & 0.04156 \\
\hline & ANOVA & n.s. & $*$ & n.s. & $* * *$ \\
\hline \multirow[t]{4}{*}{ Coding sequences excluding the homeobox } & Hox & 2.27653 & 0.18257 & 2.65921 & 0.06673 \\
\hline & Hox-derived & 5.04914 & 0.54809 & 5.26666 & 0.11320 \\
\hline & Non-Hox & 2.91160 & 0.15802 & 3.80668 & 0.04156 \\
\hline & ANOVA & n.s. & $* *$ & n.s. & $* * *$ \\
\hline \multirow[t]{4}{*}{ Coding sequences excluding repetitive regions } & Hox & 1.81997 & 0.12399 & 2.35029 & 0.05310 \\
\hline & Hox-derived & 3.71981 & 0.37759 & 4.14242 & 0.09042 \\
\hline & Non-Hox & 2.85593 & 0.15444 & 3.76458 & 0.04035 \\
\hline & ANOVA & n.s. & $*$ & n.s. & $* * *$ \\
\hline \multirow[t]{4}{*}{ Coding sequences excluding the homeobox and repetitive regions } & Hox & 1.94286 & 0.14684 & 2.33783 & 0.06146 \\
\hline & Hox-derived & 4.88928 & 0.53011 & 5.12014 & 0.11245 \\
\hline & Non-Hox & 2.85593 & 0.15444 & 3.76458 & 0.04035 \\
\hline & ANOVA & n.s. & $* *$ & n.s. & $* * *$ \\
\hline
\end{tabular}

n.s. $(\mathrm{P}>0.05), *(\mathrm{P}<0.05)$, ** $(\mathrm{P}<0.0 \mathrm{I})$, *** $(\mathrm{P}<0.00 \mathrm{I})$

tions, and reduces the difference in functional constraint. Once more, differences among groups were significant for $d_{N}(\mathrm{P}=0.030)$ and $\omega(\mathrm{P}=0.001)$. Excluding both the homeobox and the repetitive regions (bottom section of Table 1) gave intermediate results. Therefore, we can conclude that: (1)Hox and non-Hox genes are evolving similarly in terms of nucleotide substitutions, (2) Hox-derived genes are evolving much faster and with less functional constraint than the other two groups of genes, and (3) neither the homeobox nor the repetitive regions alter the estimates significantly, and thus are not entirely responsible for the two previous conclusions.

An excess of nonsynonymous over synonymous substitutions is a robust indicator of positive selection at the molecular level. Therefore, we searched for values of nonsynonymous/synonymous rate ratio $\left(d_{N} / d_{S}=\omega\right)$ greater than 1 to investigate whether Darwinian selection has been acting on any of the coding sequences analyzed in this study. However, no evidence of positive selection in any coding sequence or region of it was found.

\section{Amino acid and structural changes at the protein level}

We used the protein alignments to calculate the proportion of amino acid differences and indels. In the first case (Table 2, Figure 2), differences among the three groups -
Hox, Hox-derived and non-Hox - were not significant $(\mathrm{P}=$ $0.101)$. However, the proportion of amino acid differences was substantially higher for Hox-derived genes $(40.43 \%)$ than for Hox and non-Hox genes $(22.80 \%$ and $23.77 \%$, respectively). This result is in full agreement with our previous estimates of $d_{N}$ (Table 1 ), which showed high values of this parameter for Hox-derived genes, but very similar values for Hox and non-Hox genes.

Second, we analyzed the proportion of indels in the alignments (Table 3, Figure 2). In this case, differences among the three groups of genes were highly significant $(\mathrm{P}=$ $0.000)$. Surprisingly, differences were due to the low indel proportion in non-Hox genes $(8.73 \%)$ compared to the high values for Hox and Hox-derived genes $(25.77 \%$ and $37.53 \%$, respectively). Furthermore, we tested for differences in indel length using a nested ANOVA. The results indicated that, although the variation in indel length between genes within groups is significant $(P=0.021)$, the difference between groups is even more significant $(\mathrm{P}$ $=0.001)$. Mean indel length for Hox, Hox-derived and non-Hox genes is 4.22, 5.99 and 3.55 amino acids, respectively. Non-Hox genes not only have on average shorter indels, but also their longest indel is only 23 amino acids, in comparison with 43 and 40 amino acids for Hox and Hox-derived genes, respectively. In all groups, the indel 
(a)

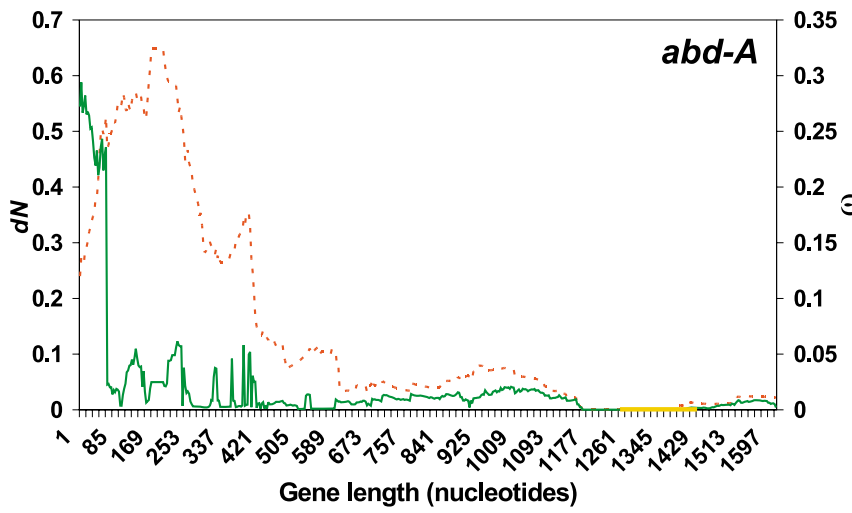

(b)

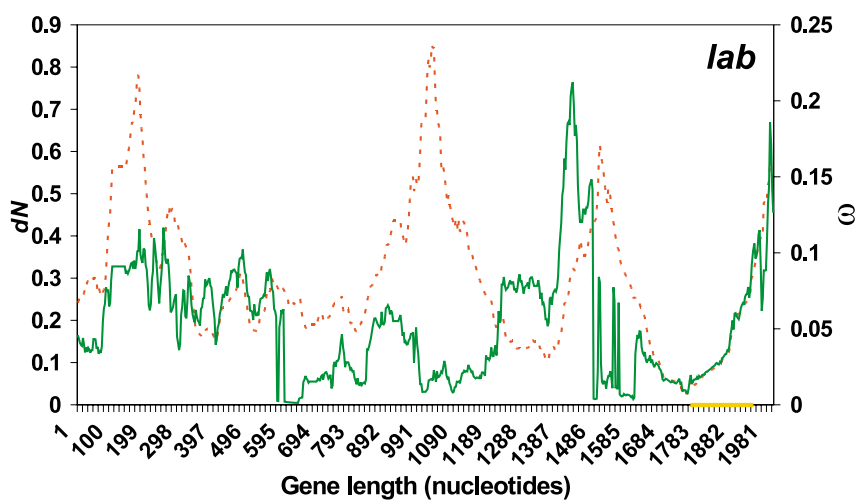

(c)

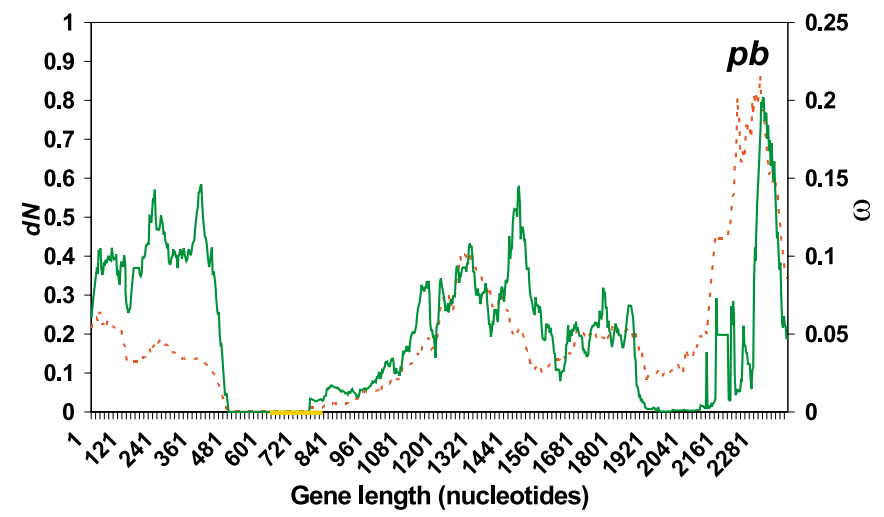

(d)

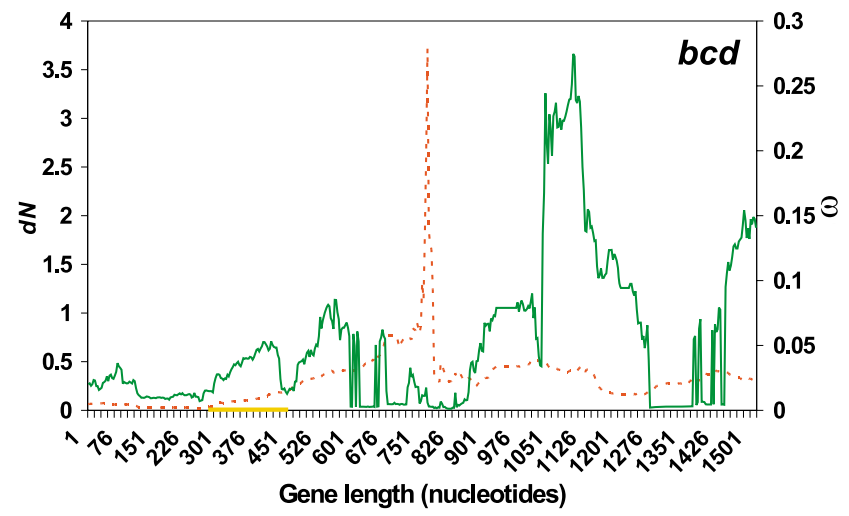

(e)

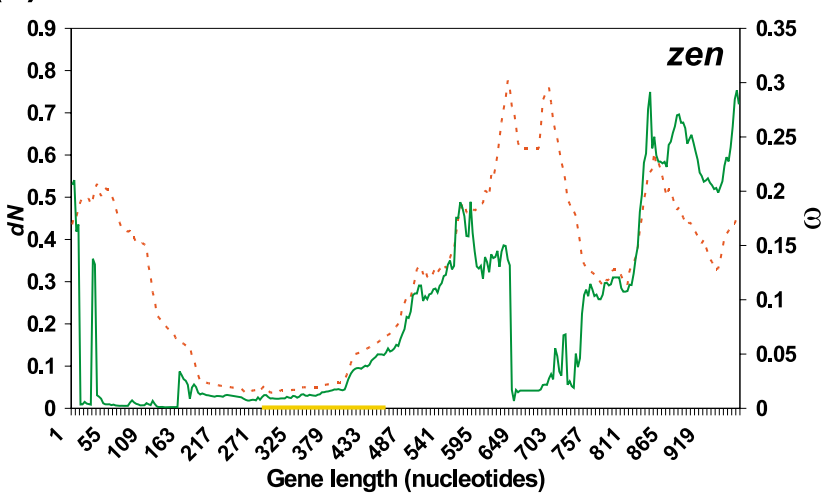

(f)

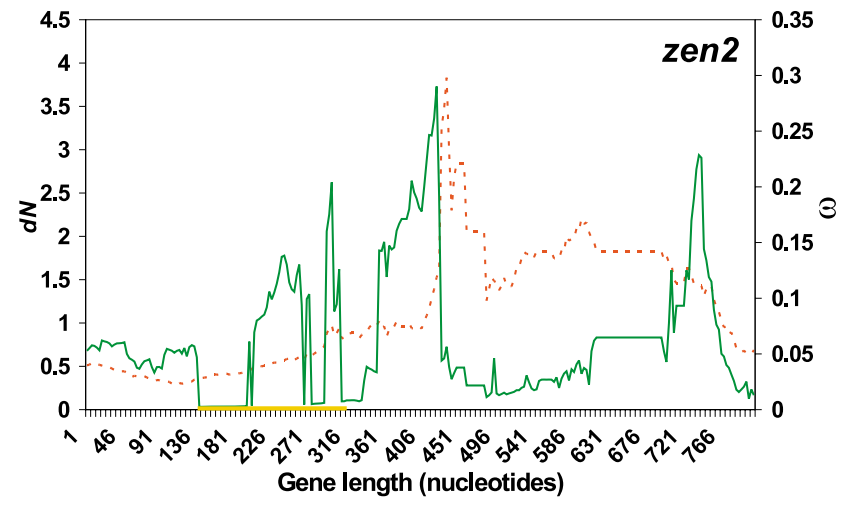

\section{Figure I}

Distribution of $d_{N}$ and $\omega$ in sliding windows along the coding sequence of genes. Distribution of $d_{N}$ (broken line) and $\omega$ (solid line) in sliding windows of 240 nucleotides. (a) $a b d-A$, (b) lab, (c) pb, (d) bcd, (e) zen and (f) zen2. In each case, the position of the homeobox is represented by a yellow box within the $X$ axis.

length distribution follows a negative exponential curve: short indels are common and their abundance declines as length increases (data not shown).
Finally, we tested whether the proportions of amino acid differences and indels are correlated. The Pearson correlation indicated that these two variables are positively but not significantly correlated $\left(\mathrm{r}_{\text {Pearson }}=0.307, \mathrm{P}=0.175\right)$. 


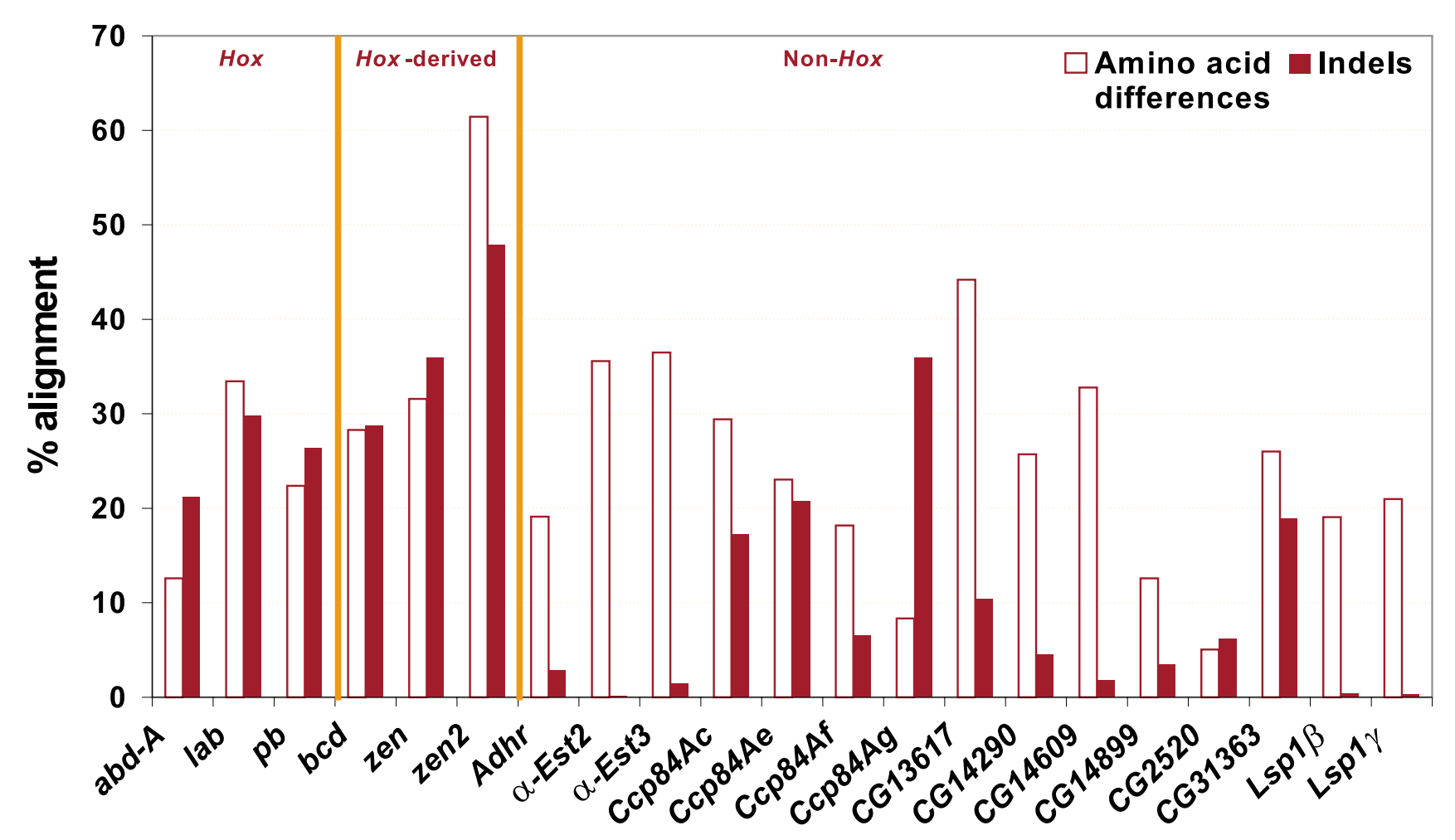

Figure 2

Proportion of amino acid differences and indels in the set of genes analyzed in this study.

Therefore, genes with a high proportion of indels do not necessarily have a high proportion of amino acid substitutions. This probably points to different causal mechanisms for amino acid substitutions and indels.

\section{Effect of long repetitive tracks in the percentages of amino acid differences and indels of Hox and Hox-derived proteins}

Most Hox and Hox-derived proteins contain large repetitive regions present throughout the protein except the region near the homeobox and other highly conserved regions (see for instance the amino acid sequence of ABD$A$ in Figure 3). Predominant repetitions are polyglutamine (poly-Q), poly-alanine (poly-A) and serinerich regions (S-rich). These repetitive regions seem to include most of the indels and amino acid differences, and therefore they might be responsible for the surprisingly high evolutionary rate of Hox and Hox-derived proteins.

To test this hypothesis, we repeated the analyses of amino acid differences and indels inside and outside these repetitive regions (see Methods), and compared these two kinds of sequences (repetitive and unique). In the case of amino acid differences (Table 2), the percentage of aligned, non-conserved amino acids is higher in repetitive regions than in unique sequence in all the three groups. The T-test for paired samples (unique versus repetitive) on proteins having both types of regions showed significant differences between unique and repetitive sequences $(\mathrm{P}=$ 0.001 ), the mean of repetitive sequences being more than twice that for unique sequences (51.01\% versus $23.19 \%$, respectively). Despite this higher percentage of amino acid differences in repetitive than in unique sequence, the three groups of genes behave in a similar manner in both types of regions (note that the ranking is the same in both unique and repetitive regions).

Finally, we wanted to determine whether or not repetitive regions accumulate a larger number of indels than unique sequence (Table 3 ). The results show that in all the three groups, the percentage of indels in repetitive regions is much higher than that in unique sequence. These differences are significant $(\mathrm{P}=0.006)$ according to a T-test for paired samples, giving an average value of $42.32 \%$ in repetitive regions versus $15.53 \%$ in unique sequence. Nevertheless, the ANOVA computed after removing repetitive regions remained highly significant $(P=0.003)$. Thus repetitive regions are not entirely responsible for the high percentage of indels in Hox and Hox-derived proteins. Therefore, Hox and Hox-derived genes have a tendency to 
Table 2: Percentage of amino acid differences in the alignment ( \pm SD) in the three groups of proteins.

\begin{tabular}{lcccc}
\hline & TOTAL & UNIQUE & REPETITIVE & T-test $\$$ \\
\hline Hox & $22.80 \pm 10.44$ & $18.22 \pm 10.50$ & $37.11 \pm 12.33$ & $* 2.97 \pm 24.08$ \\
Hox-derived & $40.43 \pm 18.26$ & $39.00 \pm 19.64$ & $55.46 \pm 31.35$ & \\
Non-Hox & $23.77 \pm 10.81$ & $23.38 \pm 10.93$ & n.s. \\
\hline ANOVA & n.s. & n.s. & \\
\hline
\end{tabular}

n.s. $(\mathrm{P}>0.05), *(\mathrm{P}<0.05)$, ** $(\mathrm{P}<0.0 \mathrm{I})$, *** $(\mathrm{P}<0.00 \mathrm{I})$.

$\S$ T-test for paired samples (unique vs. repetitive) on proteins having both types of regions [ABD-A, LAB, PB, BCD, ZEN, CcP84Ac, CGI36I7,

CGI4290 and LAP (product of CG2520)].

accumulate indels even outside of repetitive regions, which does not seem to be allowed in non-Hox genes.

\section{Discussion}

\section{Evolutionary rates of Hox genes}

This study shows that Hox genes seem to be evolving differently from other essential genes expressed in early development, with complex expression patterns or with long introns rich in cis-regulatory elements. Both the number of nonsynonymous substitutions and the degree of functional constraint are not significantly different between Hox and non-Hox genes, and this remains true even when the most peculiar regions (the homeobox and the repetitive regions) are excluded (Table 1). Therefore, Hox genes do not seem to be evolving more slowly than other non-homeotic genes, despite their essential function in the early development and even though their interchangeability between species has been proven to be functional in some cases [22-26].

Differences in the evolutionary rate among the three groups of genes (Hox, Hox-derived and non-Hox) could be mediated by some properties of genes that are correlated with the number of nucleotide substitutions $(t)$. One possibility is that Hox and Hox-derived genes experience similar background rates of mutation that are different from those of non-Hox genes. We can use the number of synonymous substitutions per synonymous site $\left(d_{S}\right)$ as a measure of the mutation rate of a gene. This variable is not significantly different among the three groups of genes ( $P$ $=0.530)$, and thus we can consider that mutation rate is constant across groups [see Additional file 2]. Another possibility is that genes within a group may have correlated levels of synonymous codon bias. Given that genes with higher codon bias tend to evolve more slowly [28,43], codon bias may contribute to spurious differences in the rates of protein evolution among groups. We have measured codon bias for each gene using the Effective Number of Codons, $N_{C}$ [44]. There are no significant differences in the codon bias among groups, and the average $N_{C}$ value for non-Hox genes is the lowest among the three groups (the highest codon bias) [see Additional files 1 and 2].

Some Hox and Hox-derived genes considered here have been included in previous studies [29,41]. Davis et al. [29] showed that the strongest negative relationship between expression profile and evolutionary rate occurs at a late stage in embryonic development, soon after the main burst of expression of segment polarity and Hox genes. However, they also show that the most constrained transcription factors and signal transducers, the functional class that contains many developmentally essential genes, are expressed precisely at the same time as the segment polarity and Hox genes. One of the two Hox genes included in their study has also been analyzed here (abd$A$ ), and it is incidentally the gene with the lowest number of nonsynonymous substitutions and the one that is most constrained in our sample of Hox genes. On the other hand, $b c d$, although being one of the first genes acting in Drosophila development, was reported in the same study

Table 3: Percentage of indels in the alignment ( \pm SD) in the three groups of proteins.

\begin{tabular}{lcccc}
\hline & TOTAL & UNIQUE & REPETITIVE & T-test\$ \\
\hline Hox & $25.77 \pm 4.31$ & $16.21 \pm 8.40$ & $44.82 \pm 2.38$ & $* 3.64 \pm 34.45$ \\
Hox-derived & $37.53 \pm 9.63$ & $34.88 \pm 12.40$ & $23.79 \pm 25.66$ & \\
Non-Hox & $8.73 \pm 10.24$ & $8.46 \pm 10.28$ & n.s. \\
\hline ANOVA & $* * *$ & $* *$ & \\
\hline
\end{tabular}

n.s. $(\mathrm{P}>0.05), *(\mathrm{P}<0.05)$, ** $(\mathrm{P}<0.01)$, *** $(\mathrm{P}<0.00 \mathrm{I})$.

$\S$ T-test for paired samples (unique vs. repetitive) on proteins having both types of regions [ABD-A, LAB, PB, BCD, ZEN, CcP84Ac, CGI36I7, CGI 4290 and LAP (product of CG2520)]. 


\begin{abstract}
Poly-Ala $50 \quad 60 \quad$ Ser-rich
Dbuz MSKFVEDSMLPKYPQFQPFISSH-LTTAPTNSS SAAVAAALAAAAASASNV-Dmel MSKFVFDSMLPKYPQFQPFISSHHLTTTPPNSS AAAVAAALAAAAASASA SVSASSSSNNNSSNTIAGSNTSNTNNSSSSPSSSSNNNSNLNLSGGSLSP

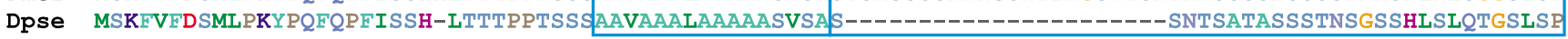

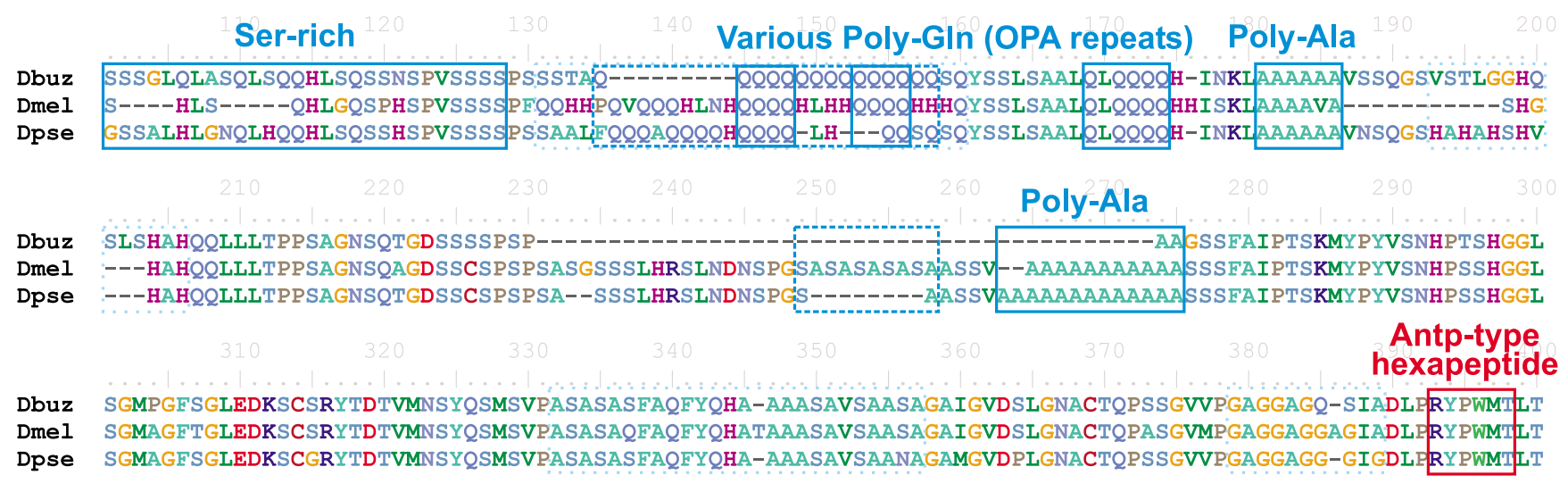

\title{
HOMEOBOX
}

Dbuz DWMGSPFERVVCGDFNGPNGC RRRGRQTYTRFQTLELEKEFHFNHYLTRRRRIEIAHALCLTERQIKIWEQNRRMKLKKEIRAVKE INEQARRDREEQE Dmel DWMGSPFERVVCGDFNGPNGC ARRRGROTYTRFOTLELEKEFHFNHYLTRRRRIEIAHALCLTEROIKIWFQNRRMKLKKEIRAVKEINEOARRDREEOE Dpse DWMGSPFERVVCGDFNGPNGCARRRGRQTYTRFQTLELEKEFHFNHYLIRRRRIEIAHALCLTERQIKIWFQNRRMKLKKEIRAVKEINEQARRDREEQE

$$
\text { Poly-GIn (OPA repeat) }
$$

\section{Poly-Arg}

Dbuz KMKAQETMKSAQQNK QVQQQQQQQQQQQQQQQQQHQQQQQQQDDHHS I ISHNP GHLHHSVV GQNDLKL GL GMG GV GV GV G GL GPGL GGGL GGNLGMMS

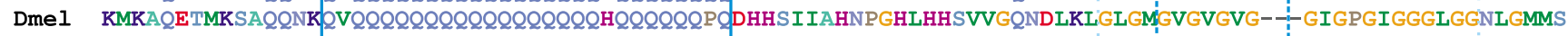
Dpse KMKAQETMKSAQQNK QVQQQQQQQQQQQQQQQ---HQQQQQQQQDHHSI ISHNPGHLHHSVVGQNDLKLGLGMGGVG---- AAGIGPGLGGGLGGLGMMS

Dbuz ALDKSNHDLLKAVSKVNS

Dmel ALDKSNHDLLKAVSKVNS

Dpse ALDKSNHDLLKAVSKVNS

\section{Figure 3}

Alignment of a Hox protein (ABD-A) showing multiple long repeats spacing functional domains. Functional domains are represented by red boxes, and repeats by blue boxes as follows: repetitive regions annotated in UniProt are represented by solid boxes, simple repeats by dashed boxes and complex repeats by dotted light boxes (see Methods). Notation: Dbuz = D. buzzatii; Dmel = D. melanogaster; Dpse $=D$. pseudoobscura.

as an exceptional case of a gene acting in the earliest stages of development but evolving surprisingly fast [29].

Furthermore, Hox genes depart from a negative correlation found in previous studies between evolutionary rate at the protein level and intron size, number of conserved noncoding sequences within introns, or regulatory complexity [32]. In this respect, all Hox genes used in this study contain a total intron size $>10 \mathrm{~Kb}$ [see Additional file 3], which corresponds to the longest intron size category used in [32]. Therefore, Hox genes are expected to evolve slowly as they contain long intronic sequences.
Both Hox-derived and non-Hox genes contain shorter intron lengths than Hox genes [see Additional file 3], and thus would be expected to evolve faster.

\section{Amino acid differences and indels}

The percentages of amino acid differences and of indels in Hox proteins also depart from the initial expectations. While the percentage of amino acid differences is not significantly different among the three groups compared (Table 2), the percentages of indels in Hox and Hoxderived proteins are much higher than that in non-Hox proteins (Table 3 ). Therefore, Hox proteins are as diver- 
gent as non-Hox proteins in terms of amino acid changes, but they are much more divergent in terms of indels. A visual inspection of the alignments pointed out a possible explanation to these results (Figure 3). Hox and some Hoxderived proteins contain large repetitive regions, mostly homopeptides, present all along the protein except the region near the homeodomain and other highly conserved regions. It is within these repetitive regions where most indels and amino acid differences seem to accumulate, in some cases resulting in poor alignment, and therefore they could be responsible for the surprisingly high amino acid and indel evolution of Hox and Hox-derived proteins.

Although repetitive regions have been shown to be richer in amino acid differences and indels than unique sequence, they do not fully explain the high variation found in Hox and Hox-derived proteins. Even excluding repetitive regions, Hox and Hox-derived genes contain many more indels than non-Hox genes, although the percentage of amino acid substitutions is not significantly different between Hox and non-Hox genes. Therefore, taking amino acid differences and indels altogether we can state that the overall rate of evolution of Hox and Hoxderived genes is faster than that of non-Hox genes. The percentage of the alignment that has changed is $43.39 \%$ in Hox proteins, $64.73 \%$ in Hox-derived proteins and $30.97 \%$ in non-Hox proteins (the percentage of amino acid differences has been recalculated before being added to the percentage of indels to account for the total number of sites, both gapped and non-gapped, in order to make both percentages comparable). Finally, a lack of correlation between the proportion of indels and amino acid differences in the set of genes used in this study highlights the different evolutionary mechanisms that regulate both types of changes.

\section{Homopeptides and other repetitions in Hox and Hox- derived proteins}

Multiple long homopeptides are found in 7\% of Drosophila proteins, most of which are essential developmental proteins expressed in the nervous system and involved in transcriptional regulation $[34,45]$. What is the role of these homopeptides? They could be tolerated, non-essential insertions that may play a role as transcriptional activity modulators. Some examples have been described in Hox and Hox-derived proteins [15] that illustrate the acquisition of new functions in the insect lineage while maintaining their homeotic role. In these examples, selection against coding changes might have been relaxed because of functional redundancy among Hox paralogs. These sequence differences could be involved in the functional divergence of Hox proteins and the evolutionary diversification of animals [15].
The large effects of Hox genes on morphology suggest that they regulate, directly or indirectly, a large number of genes. It would be expected that such pleiotropic proteins would be constrained in their sequence variation and, hence, their contribution to morphological variation. However, it has been shown that microsatellite sequences in developmental genes are a source of variation in natural populations, affecting visible traits by expanding or contracting at very high rates [46]. One intrinsic characteristic of microsatellites is their hypervariability, resulting from a balance between slippage events and point mutations $[35,36]$. Their mutation rate has been estimated to be $1.5 \times 10^{-6}$ per locus per generation in the case of trinucleotide repeats in D. melanogaster [47], and is even greater in the case of dinucleotides. These values contrast with the general mutation rate of $\sim 10^{-8}$ per site per generation of base pair substitutions [48]. These repeats typically generate regions in the alignment with high variability in sequence and length, and that are difficult to align.

A potential role for homopeptides is to serve as spacer elements between functional domains, to provide flexibility to the three-dimensional conformation, and fine-tuning domain orientation of the protein in its interactions with DNA and other proteins. To that effect, changes in nucleotide distances between target binding sites might be accompanied by complementary changes in the sequences spacing the binding domains of transcription factors (mostly homopeptides). This would produce a coordinated evolution between transcription factors and their target binding sites. Excessive expansions of homopeptides, however, have often been associated with disease in humans [49-52]. Amazingly, essential developmental proteins like homeotic proteins that apparently need such homopeptides for their correct functioning have to suffer the consequences of their quick and apparently unpredictable evolution, and sacrifice in this way the conservation that would be expected in proteins of this type.

Among non-Hox genes, the cluster of cuticular genes (Ccp84Ac, Ccp84Ae, Ccp84Af and Ccp84Ag) behave similarly to Hox and Hox-derived genes and account for the vast majority of indels in their group (Figure 2). These short proteins share a conserved C-terminal section [53] and include a 35-36 amino acid motif known as the R\&R consensus, present in many insect cuticle proteins, an extended form of which has been shown to bind chitin (chitin-bind 4; PF00379) [54]. Outside these conserved domains, cuticular proteins share hydrophobic regions dominated by tetrapeptide repeats (A-A-P-A/V), which are presumed to be functionally important $[55,56]$ and are responsible for the high percentage of indels found in these proteins. These repeats are usually complex repeats that are not annotated in UniProt, nor detected as runs of 
identical amino acid repetitions (see Methods), and thus contribute to the percentage of indels in unique sequence in non-Hox genes (Table 3 ). When complex repeats were annotated and considered as repetitive sequence (see Methods), the percentage of indels in the unique portion of all classes of genes decreased substantially, but especially in non-Hox genes [see Additional file 4]. The elimination of complex repeats in cuticular genes was crucial in this reduction, and further increased the differences among groups.

Therefore, our results show that long repetitive sequences are not enough to explain all the differences found between Hox or Hox-derived genes and non-Hox genes. Hox and Hox-derived genes have a tendency to accumulate indels outside these repetitive regions that is not observed in non-Hox genes. We propose that spontaneous deletions between short repeated sequences could be the mechanism responsible for this difference [57]. Such deletions have been described in phages [58,59], Escherichia coli $[60-65]$ and humans $[66,67]$, and predominate between short sequence similarities of as few as $5-8$ base pairs [68]. Two different models can explain the generation of spontaneous deletions: slipped mispairing during DNA synthesis, and recombination events mediated by enzymes that recognize these sequence similarities. In either case, the repetitive and compositionally biased nature of several regions within Hox and Hox-derived sequences might explain the major incidence of indels in these two groups. This would also explain the large differences in protein lengths among species that have been observed in some Hox proteins [8]. This higher probability of mutation would presumably be accompanied by a higher tolerance to indels of Hox and Hox-derived proteins outside their binding domains.

For a correct interpretation of our results, the set of nonHox genes should be an unbiased sample of genes, both in terms of protein expression and structure. We have gathered this information from the literature, and verified that our non-Hox sample comprises a variable group of genes that are expressed through the fly life cycle (from young embryo to adult) and contains a wide variety of protein domains [see Additional file 5]. Therefore, we assume that, although small, it represents an unbiased sample of all non-Hox genes in the genome, and that results presented here are reliable.

\section{The fate of Hox-derived genes after their origination by duplication}

The three Hox-derived genes used in this study $(b c d, z e n$ and zen2) originated from two consecutive duplications of the ancestral Hox3 gene. Seemingly, bcd and zen have specialized and perform separate functions in the establishment of the embryo's body plan [11-13]. This is sup- ported by our data, as these two genes have a moderate evolutionary rate but low level of functional constraint (high $d_{N} / d_{S}$ rate ratio). However, the finding of Barker $e t$ al. [42] that genes with a maternal effect experience relaxed selective constraint resulting from sex-limited expression is not supported by our data. Our results show that $b c d$ and zen are evolving at very similar rates in the Drosophila lineage, and $b c d$ is even more constrained than zen [see Additional file 1].

The function of zen 2 is unclear. It has the same expression pattern as zen and, despite its high divergence across species, it has been maintained for more than $60 \mathrm{Myr}$ [19]. Conservation of two paralogous genes maintaining the same function is unlikely, and could only be explained under some peculiar conditions (e.g. two strongly expressed genes whose products are in high demand [40]). It could be that this gene is experiencing a process of pseudogenization, supported by the fact that the evolutionary rate of zen 2 is more than twice that of $b c d$ and zen, and that it has also the highest percentage of the alignment represented by indels. If so, we would expect to see a relaxation of the functional constraint. However, the relatively high level of functional constraint of zen2 $(\omega=$ $0.09144)$ rather indicates a process of neofunctionalization, even though positive selection was not detected. The fact that this gene does not show an explicit pattern of variation of $\omega$ along its sequence (Figure 1 ) further supports the progressive loss of its original homeotic function and the acquisition of new functions.

Compared to the other two groups (Hox and non-Hox genes), Hox-derived genes are evolving significantly much faster and with less functional constraint. It is also the group with the highest proportion of amino acid differences and indels. These results reflect their relatively recent origin by duplication, which was followed by extensive changes in their role during the development of insects.

\section{Conclusion}

Many studies so far have largely focused on Hox gene homeobox sequences, and have demonstrated that they are highly conserved across species. However, Hox genes and in general all transcription factors share a particular structure where different highly conserved modules are interspersed with long repetitive regions, mostly microsatellites. Our results show that both Hox and Hox-derived genes have an overall high rate of evolution, especially in terms of indels. Moreover, although repetitive regions are richer in both amino acid differences and indels than the rest of the coding sequence, they do not seem to fully explain the differences in evolutionary rates found between Hox or Hox-derived genes and non-Hox genes. Therefore, by using complete gene sequences rather than 
their conserved modules, we observe that the Hox gene evolutionary rate is as high as that of non-Hox genes in terms of nucleotide evolution, and even higher in terms of indels. Hox-derived genes constitute the group with the highest evolutionary rate by all criteria. These results emphasize the need to take into account indels in addition to nucleotide substitutions in order to estimate evolutionary rates accurately. This study is the first quantification of the rates of nucleotide and indel evolution in these groups of genes, and shows that Hox and Hox-derived genes have a higher evolutionary dynamics than other developmental genes.

\section{Methods}

\section{Genes analyzed and their classification}

All the completely sequenced genes in D. buzzatii with a clear ortholog in D. melanogaster and D. pseudoobscura (23) were included in our analysis: $a b d-A, l a b, p b, b c d, z e n$, zen2, Dbuz|Ccp3 (ortholog of Dmel|Ccp84Ac), Dbuz|Ccp6 (ortholog of Dmel|Ccp84Ae), Dbuz|Ccp7 (ortholog of Dmel|Ccp84Af), Dbuz|Ccp8 (ortholog of Dmel|Ccp84Ag), CG1288, CG14290, CG14609, CG14899, CG17836, CG2520 and CG31363 from Negre et al. [19]; Adh-related (Adhr) from Betran and Ashburner [69]; $\alpha$-Esterase-2 ( $\alpha$ Est2) and $\alpha$-Esterase-3 ( $\alpha$-Est3) from Robin et al. [70]; CG13617 from Puig, Caceres, and Ruiz [71]; and Larval serum protein $1 \beta(L s p 1 \beta)$ and $L s p 1 \gamma$ from Gonzalez, Casals and Ruiz [72]. Sequences of D. melanogaster orthologs were collected from Flybase $[73,74]$, and those of D. pseudoobscura were annotated on the scaffolds from the whole genome shotgun sequencing project $[75,76]$. We identified the $D$. pseudoobscura orthologs by using the alignment of this species with the D. melanogaster genome generated by the Berkeley Genome Pipeline [77], and annotated the target sequences with the aid of ARTEMIS v. 7 [78] and BIOEDIT v. 7.0.4.1 [79]. A complete list of all genes, accession numbers (from Genbank or Flybase) and chromosomal locations is provided [see Additional file 3]. The longest transcript of each gene was used for the analyses. Genes were classified into three categories: 1) Hox genes $(a b d-A$, lab and $p b) ; 2)$ Hox-derived genes ( $b c d$, zen and zen 2 ); and 3) non-Hox genes (the remaining 17 genes). Results in each group were produced by calculating the average of all the genes within the group.

\section{Sequence annotation and alignment}

A set of Perl scripts, together with modules from PDA v. 1.4 [80] and BIOPERL v. 1.2.3 [81], were used to automatically check sequence annotations, extract the coding sequences (CDSs) of the selected transcripts and calculate basic gene structure and base composition parameters (gene and protein lengths; codon bias measured by the Effective Number of Codons $\left(N_{C}\right)$; and $\mathrm{G}+\mathrm{C}$ content in second, third and all codon positions) [see Additional file $1]$. Differences among the three groups of genes were tested with one-way ANOVAs and pairwise contrast tests [82], assuming homogeneity of variances for those variables that gave non-significant $P$ values for the Levene test [83] [see Additional file 2]. Orthologous coding sequences in D. buzzatii, D. melanogaster and D. pseudoobscura were aligned according to their translation to protein using RevTrans 1.3 Server [84] with some manual editing using BIOEDIT v. 7.0.4.1 [79]. Two non-Hox genes of the initial sample (CG1288 and CG17836) showed a doubtful alignment, containing many gaps and few residue matches, and thus were excluded from the analyses to avoid unreliable estimates. A total of 15 non-Hox genes were therefore used in this study.

\section{Estimation of evolutionary rates}

The numbers of synonymous and nonsynonymous substitutions per site ( $d_{S}$ and $d_{N^{\prime}}$ respectively) were estimated on the nucleotide alignments of each gene using maximum likelihood methods with the program codeml of the PAML v. 3.14 package [85] [see Additional file 1]. We used an unrooted tree and the codon equilibrium frequencies $\left(\pi_{i}\right)$ estimated from the nucleotide frequencies of the three codon sites (F3X4 option of codeml). Differences among the three groups of genes were tested using one-way ANOVAs and pairwise contrast tests as before. Furthermore, we visualized differences along the genes by plotting $d_{N}$ and $\omega$ in sliding windows of 240 nucleotides and a step size of three nucleotides (one codon).

\section{Measurement of amino acid differences and indels}

We measured the proportion of amino acid differences and indels in the protein alignments (translated from the previous nucleotide alignments) using in-house Perl scripts. The methodology was based on measuring the number of non-conserved positions due to either amino acid differences (point changes) or indels (structural changes) in the protein multiple alignments (e.g. the minimum indel length is one amino acid, corresponding to three nucleotides in the nucleotide sequence). We can estimate in this way the percentage of the protein which has been changed in our set of species. We think that this is a simple (yet somewhat rough) measure to estimate the degree of constraint relaxation of proteins.

Specifically, the number of amino acid differences was computed as the number of non-gapped positions with non-identical amino acids in the three species. All percentages are given in relation to the total number of aligned amino acids (non-gapped positions). Similarly, the number of indels was computed as the number of different indels (gaps affecting different positions) in the complete alignment (gapped and non-gapped sites). Therefore, an indel shared by two species was considered a single indel, while overlapping gaps were considered separately. Indel lengths were taken into account to calcu- 
late the percentage of the alignment affected by indels. In this case, all percentages are given in relation to the total length of the alignment (gapped and non-gapped positions).

We used one-way ANOVAs to test for differences between Hox, Hox-derived and non-Hox proteins in both parameters: the proportion of amino acid differences and the proportion of indels. We also used the Pearson correlation coefficient to test for a correlation between the two measures (e.g. to test whether proteins with a high proportion of amino acid differences also have a high proportion of indels), and a nested ANOVA [82] to test for differences in indel length among the three groups, taking into account the variation within groups.

\section{Contribution of the homeobox and the repetitive regions to the evolutionary rates}

In order to test the effect of the homeobox and the repetitive regions in our estimates of nucleotide substitutions, we repeated the previous analyses excluding one or both types of sequence. Repetitive regions were identified in three different ways. First, we searched in the UniProt Knowledgebase Release 8.6 (Swiss-Prot Release $50.6+$ TrEMBL Release 33.6) [86] for annotated compositionally biased regions (defined in the feature table as COMPBIAS) in the protein sequences encoded by Hox, Hoxderived and non-Hox genes [see Additional file 3]. In the case of Hox genes, all three genes in the group contained at least one annotated repetitive region, while for Hoxderived and non-Hox genes only one entry of each group (bcd and CG2520, respectively) contained annotated repetitive regions. Note that only repeats in $D$. melanogaster are identified by using this methodology. Second, we identified simple repeats as those runs of 5 or more identical amino acids (e.g. QQQQQ), or at least 4 identical repetitions of 2 or more amino acids (e.g. GVGVGVGV), in any of the three species. By using this second approach, we extended the number of proteins with repetitive sequences in both the Hox-derived and non-Hox groups. Finally, we tried to visually annotate complex repeats as those imperfect runs of amino acid repetitions or compositionally biased regions in the protein (e.g. regions in the protein with a high content of $\mathrm{Q}$, $\mathrm{S}, \mathrm{A}, \mathrm{P}, \mathrm{H}, \mathrm{G}, \mathrm{V}$, etc.). Data was analyzed using a combination of the three approaches as follows: (1) using UniProt only; (2) using UniProt + Simple repeats; and (3) using UniProt + Simple repeats + Complex repeats. Because the identification of complex repeats is somewhat subjective, we present in the main text the results obtained by identifying repeats using the second combination (UniProt + Simple repeats). However, results do not differ significantly among the three combinations [see Additional file $4]$.
We also calculated the proportion of amino acid differences and indels in repetitive and non-repetitive (unique) sequence in the three groups, and tested for differences between these two types of regions using a T-test for paired samples [82] on those proteins having both types of regions.

\section{Abbreviations}

$t=$ number of nucleotide substitutions per codon; $d_{S}=$ number of synonymous substitutions per synonymous site; $d_{N}=$ number of nonsynonymous substitutions per nonsynonymous site; $\omega=d_{N} / d_{S}$ ratio that measures the level of functional constraint; $\kappa=$ transition/transversion rate ratio; $N_{C}=$ Effective Number of Codons.

\section{Authors' contributions}

SC carried out the analyses and drafted the manuscript. $\mathrm{BN}$ participated in obtaining the data and in the design of the analyses. AB participated in the statistical analysis. AR conceived the study, and participated in its design and coordination. All authors read and approved the final manuscript.

\section{Additional material}

\author{
Additional File 1 \\ Parameters of gene structure, base composition and nucleotide evolution \\ for each gene. \\ Click here for file \\ [http://www.biomedcentral.com/content/supplementary/1471- \\ 2148-6-106-S1.pdf] \\ Additional File 2 \\ ANOVA and contrast analyses for all group comparisons. \\ Click here for file \\ [http://www.biomedcentral.com/content/supplementary/1471- \\ 2148-6-106-S2.pdf]

\section{Additional File 3} \\ Genes from D. buzzatii, D. melanogaster and D. pseudoobscura used \\ in the analyses with their accession number in Genbank or Flybase and \\ their location on the chromosome. \\ Click here for file \\ [http://www.biomedcentral.com/content/supplementary/1471- \\ 2148-6-106-S3.pdf]
}




\section{Additional File 4 \\ Set of tables of the main text, obtained according to three different anno- tation criteria to define repetitive sequences. \\ Click here for file \\ [http://www.biomedcentral.com/content/supplementary/1471- 2148-6-106-S4.pdf] \\ Additional File 5 \\ Structure and expression of non-Hox proteins. \\ Click here for file \\ [http://www.biomedcentral.com/content/supplementary/1471- 2148-6-106-S5.pdf]}

\section{Acknowledgements}

The authors would like to thank Natalia Petit for helpful discussions, and Kristen Panfilio and two anonymous referees for valuable comments on this manuscript. This work has been supported by grant BMC2002-01708 from the Dirección General de Enseñanza Superior e Investigación Científica (MEC, Spain) awarded to AR, a doctoral FPI fellowship from the Ministerio de Ciencia y Tecnología (BES-2003-04I6) awarded to SC and a doctoral Fl/ DGR fellowship from the Generalitat de Catalunya awarded to BN.

\section{References}

I. Carroll SB, Grenier JK, Weatherbee SD: From DNA to diversity: Molecular Genetics and Evolution of Animal Design. 2nd ed. edition. Blackwell; 2005.

2. Lewis EB: A gene complex controlling segmentation in Drosophila. Nature 1978, 276(5688):565-570.

3. Kaufman TC, Lewis R, Wakimoto B: Cytogenetic Analysis of Chromosome 3 in Drosophila melanogaster: the Homeotic Gene Complex in Polytene Chromosome Interval 84A-B. Genetics 1980, 94(I): I I5-133.

4. Cook CE, Smith ML, Telford MJ, Bastianello A, Akam M: Hox genes and the phylogeny of the arthropods. Curr Biol 200I, I I(I 0):759-763.

5. Hughes CL, Kaufman TC: Hox genes and the evolution of the arthropod body plan. Evol Dev 2002, 4(6):459-499.

6. Hughes CL, Liu PZ, Kaufman TC: Expression patterns of the rogue Hox genes Hox3/zen and fushi tarazu in the apterygote insect Thermobia domestica. Evol Dev 2004, 6(6):393-40I.

7. Lewis EB, Pfeiffer BD, Mathog DR, Celniker SE: Evolution of the homeobox complex in the Diptera. Curr Biol 2003, I 3( I 5):R587-8.

8. Negre B, Ranz JM, Casals F, Caceres M, Ruiz A: A new split of the Hox gene complex in Drosophila: relocation and evolution of the gene labial. Mol Biol Evol 2003, 20( I 2):2042-2054.

9. Von Allmen G, Hogga I, Spierer A, Karch F, Bender W, Gyurkovics H, Lewis E: Splits in fruitfly Hox gene complexes. Nature 1996, 380(6570): I I6.

10. Negre B, Ruiz A: HOM-C evolution in Drosophila: is there a need for Hox gene clustering? Trends Genet 2007 in press.

II. Bonneton F: [Extreme divergence of a homeotic gene: the bicoid case]. Med Sci (Paris) 2003, 19:1265-1270.

12. Stauber M, Jackle H, Schmidt-Ott U: The anterior determinant bicoid of Drosophila is a derived Hox class 3 gene. Proc Natl Acad Sci U S A 1999, 96(7):3786-3789.

13. Stauber M, Prell A, Schmidt-Ott U: A single Hox3 gene with composite bicoid and zerknullt expression characteristics in nonCyclorrhaphan flies. Proc Natl Acad Sci U S A 2002, 99( I):274-279.

14. Telford MJ: Evidence for the derivation of the Drosophila fushi tarazu gene from a Hox gene orthologous to lophotrochozoan Lox5. Curr Biol 2000, I0(6):349-352.
15. Galant R, Carroll SB: Evolution of a transcriptional repression domain in an insect Hox protein. Nature 2002, 4I5(6874):910-913.

16. Shiga $Y$, Yasumoto $R$, Yamagata $H$, Hayashi $S$ : Evolving role of Antennapedia protein in arthropod limb patterning. Development 2002, I 29(I5):3555-356I.

17. Lohr U, Yussa M, Pick L: Drosophila fushi tarazu. a gene on the border of homeotic function. Curr Biol 200 I, I I ( I 8): | 403-I4I2.

18. Akam M, Averof M, Castelli-Gair J, Dawes R, Falciani F, Ferrier D: The evolving role of Hox genes in arthropods. Dev Suppl 1994:209-2I5.

19. Negre B, Casillas S, Suzanne M, Sanchez-Herrero E, Akam M, Nefe$\operatorname{dov} M$, Barbadilla $A$, de Jong $P$, Ruiz $A$ : Conservation of regulatory sequences and gene expression patterns in the disintegrating Drosophila Hox gene complex. Genome Res 2005, I 5(5):692-700.

20. Berleth T, Burri M, Thoma G, Bopp D, Richstein S, Frigerio G, Noll $M$, Nusslein-Volhard C: The role of localization of bicoid RNA in organizing the anterior pattern of the Drosophila embryo. Embo J 1988, 7(6): I749-1756.

21. Rushlow C, Doyle H, Hoey T, Levine M: Molecular characterization of the zerknullt region of the Antennapedia gene complex in Drosophila. Genes Dev 1987, I(1 0): I 268-1279.

22. McGinnis N, Kuziora MA, McGinnis W: Human Hox-4.2 and Drosophila deformed encode similar regulatory specificities in Drosophila embryos and larvae. Cell 1990, 63(5):969-976.

23. Zhao JJ, Lazzarini RA, Pick L: The mouse Hox-I.3 gene is functionally equivalent to the Drosophila Sex combs reduced gene. Genes Dev 1993, 7(3):343-354.

24. Bachiller D, Macias A, Duboule D, Morata G: Conservation of a functional hierarchy between mammalian and insect Hox/ HOM genes. Embo J 1994, I3(8): 1930-194I.

25. Zakany J, Gerard M, Favier B, Potter SS, Duboule D: Functional equivalence and rescue among group I I Hox gene products in vertebral patterning. Dev Biol 1996, I 76(2):325-328.

26. Greer JM, Puetz J, Thomas KR, Capecchi MR: Maintenance of functional equivalence during paralogous Hox gene evolution. Nature 2000, 403(6770):661-665.

27. Drummond DA, Bloom JD, Adami C, Wilke CO, Arnold FH: Why highly expressed proteins evolve slowly. Proc Natl Acad Sci U S A 2005, I 02(40): |4338-14343.

28. Pal C, Papp B, Hurst LD: Highly expressed genes in yeast evolve slowly. Genetics 200I, I58(2):927-931.

29. Davis JC, Brandman O, Petrov DA: Protein evolution in the context of Drosophila development. J Mol Evol 2005, 60(6):774-785.

30. Powell JR, Caccone A, Gleason JM, Nigro L: Rates of DNA evolution in Drosophila depend on function and developmental stage of expression. Genetics 1993, I33(2):291-298.

31. Riedl R: Order in living organisms: A systems analysis of evolution. New York, Wiley; 1978.

32. Marais G, Nouvellet P, Keightley PD, Charlesworth B: Intron size and exon evolution in Drosophila. Genetics 2005, I 70(I):48I-485.

33. Averof $M$ : Arthropod Hox genes: insights on the evolutionary forces that shape gene functions. Curr Opin Genet Dev 2002, I 2(4):386-392.

34. Karlin S, Burge C: Trinucleotide repeats and long homopeptides in genes and proteins associated with nervous system disease and development. Proc Natl Acad Sci U S A 1996, 93(4): I560- I565.

35. Ellegren H: Microsatellites: simple sequences with complex evolution. Nat Rev Genet 2004, 5(6):435-445.

36. Kruglyak S, Durrett RT, Schug MD, Aquadro CF: Equilibrium distributions of microsatellite repeat length resulting from a balance between slippage events and point mutations. Proc Natl Acad Sci U S A 1998, 95( 1 8): 10774-10778.

37. Lynch M, Force A: The probability of duplicate gene preservation by subfunctionalization. Genetics 2000, I 54(I):459-473.

38. Lynch M, Conery JS: The evolutionary fate and consequences of duplicate genes. Science 2000, 290(5494): I I I I- I I 55.

39. Long M, Betran E, Thornton K, Wang W: The origin of new genes: glimpses from the young and old. Nat Rev Genet 2003, 4(I I):865-875.

40. Zhang J: Evolution by gene duplication: an update. Trends Ecol Evol 2003, I 8(6):292-298. 
4I. Baines JF, Chen Y, Das A, Stephan W: DNA sequence variation at a duplicated gene: excess of replacement polymorphism and extensive haplotype structure in the Drosophila melanogaster bicoid region. Mol Biol Evol 2002, I 9(7):989-998.

42. Barker MS, Demuth JP, Wade MJ: Maternal Expression Relaxes Constraint on Innovation of the Anterior Determinant, bicoid. PLoS Genet 2005, I(5):e57.

43. Akashi $\mathrm{H}$ : Gene expression and molecular evolution. Curr Opin Genet Dev 200I, I I(6):660-666.

44. Wright $F$ : The 'effective number of codons' used in a gene. Gene 1990, 87(I):23-29.

45. Karlin S, Brocchieri L, Bergman A, Mrazek J, Gentles A): Amino acid runs in eukaryotic proteomes and disease associations. Proc Natl Acad Sci U S A 2002, 99(I):333-338.

46. Fondon JW 3rd, Garner HR: Molecular origins of rapid and continuous morphological evolution. Proc Natl Acad Sci U S A 2004, I0I(52): I 8058- I8063.

47. Schug MD, Hutter CM, Wetterstrand KA, Gaudette MS, Mackay TF, Aquadro CF: The mutation rates of di-, tri- and tetranucleotide repeats in Drosophila melanogaster. Mol Biol Evol 1998, I5(I2): I75I-1760.

48. Li WH: Molecular Evolution. Sunderland Massachusetts, Sinauer Associates, Inc.; 1997.

49. Hancock JM, Worthey EA, Santibanez-Koref MF: A role for selection in regulating the evolutionary emergence of diseasecausing and other coding CAG repeats in humans and mice. Mol Biol Evol 200I, I8(6): 1014-1023.

50. Karlin S, Chen C, Gentles AJ, Cleary M: Associations between human disease genes and overlapping gene groups and multiple amino acid runs. Proc Natl Acad Sci U S A 2002, 99(26): $17008-17013$

5I. Albrecht A, Mundlos S: The other trinucleotide repeat: polyalanine expansion disorders. Curr Opin Genet Dev 2005, I 5(3):285-293.

52. Brown LY, Brown SA: Alanine tracts: the expanding story of human illness and trinucleotide repeats. Trends Genet 2004, 20(I):5I-58

53. Rebers JE, Riddiford LM: Structure and expression of a Manduca sexta larval cuticle gene homologous to Drosophila cuticle genes. J Mol Biol 1988, 203(2):4I I-423.

54. Rebers JE, Willis JH: A conserved domain in arthropod cuticular proteins binds chitin. Insect Biochem Mol Biol 200I, 3 I (II): 1083-1093.

55. Talbo G, Hojrup P, Rahbek-Nielsen H, Andersen SO, Roepstorff $P$ : Determination of the covalent structure of an $\mathrm{N}$ - and C-terminally blocked glycoprotein from endocuticle of Locusta migratoria. Combined use of plasma desorption mass spectrometry and Edman degradation to study post-translationally modified proteins. Eur J Biochem 1991, 195(2):495-504.

56. Andersen SO, Rafn K, Roepstorff P: Sequence studies of proteins from larval and pupal cuticle of the yellow meal worm, Tenebrio molitor. Insect Biochem Mol Biol 1997, 27(2): I2I-131.

57. Albertini AM, Hofer M, Calos MP, Miller JH: On the formation of spontaneous deletions: the importance of short sequence homologies in the generation of large deletions. Cell 1982, 29(2):319-328.

58. Studier FW, Rosenberg AH, Simon MN, Dunn J]: Genetic and physical mapping in the early region of bacteriophage T7 DNA. I Mol Biol 1979, I 35(4):917-937.

59. Pribnow D, Sigurdson DC, Gold L, Singer BS, Napoli C, Brosius J, Dull T], Noller HF: rll cistrons of bacteriophage T4. DNA sequence around the intercistronic divide and positions of genetic landmarks. J Mol Biol I 98I, I 49(3):337-376.

60. Brake AJ, Fowler AV, Zabin I, Kania J, Muller-Hill B: beta-Galactosidase chimeras: primary structure of a lac repressor-betagalactosidase protein. Proc Natl Acad Sci U S A 1978, 75(1 0):4824-4827.

61. Fedoroff NV: Deletion mutants of Xenopus laevis $5 \mathrm{~S}$ ribosomal DNA. Cell 1979, 16(3):55|-563.

62. Ghosal D, Saedler $\mathrm{H}$ : IS2-6I and IS2-6II arise by illegitimate recombination from IS2-6. Mol Gen Genet 1979, I 76(2233-238 [http://www.springerlink.com/content/u340577860368426/].

63. Post LE, Arfsten AE, Davis GR, Nomura M: DNA sequence of the promoter region for the alpha ribosomal protein operon in Escherichia coli. J Biol Chem 1980, 255( I 0):4653-4659.
64. Ross DG, Swan J, Kleckner N: Nearly precise excision: a new type of DNA alteration associated with the translocatable element Tn I0. Cell 1979, 16(4):733-738.

65. Wu AM, Chapman AB, Platt T, Guarente LP, Beckwith J: Deletions of distal sequence after termination of transcription at the end of the tryptophan operon in E. coli. Cell 1980 , 19(4):829-836.

66. Efstratiadis A, Posakony JW, Maniatis T, Lawn RM, O'Connell C, Spritz RA, DeRiel JK, Forget BG, Weissman SM, Slightom JL, Blechl $A E$, Smithies O, Baralle FE, Shoulders CC, Proudfoot NJ: The structure and evolution of the human beta-globin gene family. Cell 1980, 2 1(3):653-668

67. Marotta CA, Wilson JT, Forget BG, Weissman SM: Human betaglobin messenger RNA. III. Nucleotide sequences derived from complementary DNA. I Biol Chem 1977, 252(14):5040-5053.

68. Farabaugh PJ: Sequence of the lacl gene. Nature 1978 , 274(5673): 765-769.

69. Betran E, Ashburner M: Duplication, dicistronic transcription, and subsequent evolution of the Alcohol dehydrogenase and Alcohol dehydrogenase-related genes in Drosophila. Mol Biol Evol 2000, 17(9): 1344-1352.

70. Robin GC, Russell RJ, Cutler DJ, Oakeshott JG: The evolution of an alpha-esterase pseudogene inactivated in the Drosophila melanogaster lineage. Mol Biol Evol 2000, I 7(4):563-575.

71. Puig M, Caceres M, Ruiz A: Silencing of a gene adjacent to the breakpoint of a widespread Drosophila inversion by a transposon-induced antisense RNA. Proc Natl Acad Sci U S A 2004 I0I(24):90|3-9018.

72. Gonzalez J, Casals F, Ruiz A: Duplicative and conservative transpositions of larval serum protein I genes in the genus Drosophila. Genetics 2004, 168(1):253-264.

73. Drysdale RA, Crosby MA, FlyBase Consortium: FlyBase: genes and gene models. Nucleic Acids Res 2005, 33:D390-5.

74. Celniker SE, Wheeler DA, Kronmiller B, Carlson JW, Halpern A, Patel S, Adams M, Champe M, Dugan SP, Frise E, Hodgson A, George RA, Hoskins RA, Laverty T, Muzny DM, Nelson CR, Pacleb JM, Park S, Pfeiffer BD, Richards S, Sodergren EJ, Svirskas R, Tabor PE, Wan K, Stapleton M, Sutton GG, Venter C, Weinstock G, Scherer SE, Myers EW, Gibbs RA, Rubin GM: Finishing a whole-genome shotgun: release 3 of the Drosophila melanogaster euchromatic genome sequence. Genome Biol 2002, 3(I 2):RESEARCH0079.

75. Richards S, Liu Y, Bettencourt BR, Hradecky P, Letovsky S, Nielsen R, Thornton K, Hubisz MJ, Chen R, Meisel RP, Couronne O, Hua S, Smith MA, Zhang P, Liu J, Bussemaker HI, van Batenburg MF, Howells SL, Scherer SE, Sodergren E, Matthews BB, Crosby MA, Schroeder AJ, Ortiz-Barrientos D, Rives CM, Metzker ML, Muzny DM, Scott G, Steffen D, Wheeler DA, Worley KC, Havlak P, Durbin KJ, Egan A, Gill R, Hume J, Morgan MB, Miner G, Hamilton C, Huang Y, Waldron L, Verduzco D, Clerc-Blankenburg KP, Dubchak I, Noor MA, Anderson W, White KP, Clark AG, Schaeffer SW, Gelbart W, Weinstock GM, Gibbs RA: Comparative genome sequencing of Drosophila pseudoobscura: chromosomal, gene, and cis-element evolution. Genome Res 2005, I 5(I): I-18.

76. Benson DA, Karsch-Mizrachi I, Lipman DJ, Ostell J, Wheeler DL: GenBank. Nucleic Acids Res 2006, 34:DI6-20.

77. Mayor C, Brudno M, Schwartz JR, Poliakov A, Rubin EM, Frazer KA, Pachter LS, Dubchak I: VISTA : visualizing global DNA sequence alignments of arbitrary length. Bioinformatics 2000, I6( I I): 1046-1047.

78. Berriman $M$, Rutherford $K$ : Viewing and annotating sequence data with Artemis. Brief Bioinform 2003, 4(2): 124-132.

79. Hall TA: BioEdit: a user-friendly biological sequence alignment editor and analysis program for Windows 95/98/NT. Nucl Acids Symp Ser 1999, 41:95-98.

80. Casillas S, Barbadilla A: PDA: a pipeline to explore and estimate polymorphism in large DNA databases. Nucleic Acids Res 2004, 32(Web Server issue):WI66-9.

8I. Stajich JE, Block D, Boulez K, Brenner SE, Chervitz SA, Dagdigian C, Fuellen G, Gilbert JG, Korf I, Lapp H, Lehvaslaiho H, Matsalla C, Mungall CJ, Osborne BI, Pocock MR, Schattner P, Senger M, Stein LD, Stupka E, Wilkinson MD, Birney E: The Bioperl toolkit: Perl modules for the life sciences. Genome Res 2002, I2(10):|6||-16|8.

82. Sokal RR, Rohlf FJ: Biometry: The principles and practice of statistics in biological research. New York, W.H. Freeman and Co.; 1995. 
83. Levene H: Contributions to Probability and Statistics: Essays in Honor of Harold Hotelling. Volume I. Edited by: al. O. Stanford University Press; 1960:278-292.

84. Wernersson R, Pedersen AG: RevTrans: Multiple alignment of coding DNA from aligned amino acid sequences. Nucleic Acids Res 2003, 3 I (I3):3537-3539.

85. Yang Z: PAML: a program package for phylogenetic analysis by maximum likelihood. Comput Appl Biosci 1997, 13(5555-556 [http://bioinformatics.oxfordjournals.org/cgi/reprint//3/5/555].

86. Bairoch A, Apweiler R, Wu CH, Barker WC, Boeckmann B, Ferro S, Gasteiger E, Huang H, Lopez R, Magrane M, Martin MJ, Natale DA, O'Donovan C, Redaschi N, Yeh LS: The Universal Protein Resource (UniProt). Nucleic Acids Res 2005, 33(Database issue):DI54-9.

Publish with Bio Med Central and every scientist can read your work free of charge

"BioMed Central will be the most significant development for disseminating the results of biomedical research in our lifetime. "

Sir Paul Nurse, Cancer Research UK

Your research papers will be:

- available free of charge to the entire biomedical community

- peer reviewed and published immediately upon acceptance

- cited in PubMed and archived on PubMed Central

- yours - you keep the copyright

Submit your manuscript here:

http://www.biomedcentral.com/info/publishing_adv.asp
BioMedcentral 